\title{
Asthma - Geschichtliche Entwicklung, Status quo und Ausblick
}

\author{
Asthma - Historical Development, Current Status and Perspectives
}

Autor

Institut

\section{J. C. Virchow}

Universitätsklinikum Rostock, Klinik und Poliklinik für Innere Medizin eingereicht 25.7. 2010

akzeptiert nach Revision 27. 7.2010

\section{Bibliografie}

DOI http://dx.doi.org/

10.1055/s-0030-1255695

Pneumologie 2010; 64:

541-549 @ Georg Thieme

Verlag KG Stuttgart - New York

ISSN 0934-8387

\section{Korrespondenzadresse}

Prof. Dr. med. J. C. Virchow Jr

Universitätsklinikum Rostock

Klinik und Poliklinik

für Innere Medizin

Ernst-Heydemann-Str. 6

18055 Rostock

j.c.virchow@med.uni-rostock.de

\section{Zusammenfassung \\ $\nabla$}

Asthma ist keine neue, aber eine der häufigsten chronischen Erkrankungen; schätzungsweise 45\% der Erwachsenen und mehr als 10\% der Kinder in Deutschland sind betroffen, was Asthma zu einer der wichtigsten Volkskrankheiten macht. Im Laufe der letzten hundert Jahre haben sich die Vorstellungen zur Pathogenese von einer neurotischen Erkrankung über eine Erkrankung der glatten Muskulatur oder der Mastzelle oder spezieller Mediatoren wie dem Platelet activating factor bzw. einer Erkrankung durch Eosinophile bzw. deren regulierende T-Zellen zu einer komplexen Störung des adaptiven Immunsystems gewandelt. Auch die Therapie unterlag einer insgesamt dramatischen Änderung: Während Asthma vor 30 Jahren noch als Anfallskrankheit häufig, vor allem des Nachts, ärztlicher Interventionen bedurfte und Patienten im Status asthmaticus entweder verstarben oder invasiv beatmet werden mussten, hat sich dies heute durch den Einsatz moderner Asthmatherapie deutlich gewandelt. Dennoch ist Asthma auch heute noch, selbst unter wirksamer Therapie, keine Bagetell-Erkrankung und Patienten sind auch unter Therapie einer erheblichen Morbidität ausgesetzt, deren weitere Verbesserung anzustreben ist. Die Entwicklungen der letzten hundert Jahre werden im Folgenden, wenn auch oft aus der besser wissenden Retrospektive betrachtet, wobei der Status quo beleuchtet und sogar ein Ausblick (ohne Gewähr) in die Zukunft gewagt wird.

\section{Abstract \\ $\nabla$}

Asthma is not a new disease. It is one of the most common chronic disorders affecting approximately $4-5 \%$ of adults and more than $10 \%$ of children in Germany. This turns asthma into one of the most prevalent chronic disorders. Over the last century ideas about its pathogenesis have changed many times. While around one hundred years ago asthma was often considered a neurotic disease, changes in airway smooth muscle, mast cell accumulation and activation or specific mediators such as platelet-activating factor have since been incriminated in its pathogenesis. Eosinophils, cytokines and T-lymphocytes were favourites some time later. Nowadays, - and this is unlikely to be the end of the story - asthma is considered as a complex disorder of the adaptive immune system. Therapeutic approaches have changed dramatically, too. While until about 30 years ago asthma was still considered a Smooth muscle disorder, recurrent attacks of asthma which required frequent, mostly nocturnal interventions, status asthmaticus, or the necessity of mechanical, invasive ventilation have markedly decreased. In view of the asthma epidemic in recent years, this development suggests that current treatments are at least partially effective. In spite of this patients with asthma are often only moderately well controlled with considerable morbidity from the disease as well as its treatment. Thus, despite recent advances in diagnosis and treatment, asthma is still not a trivial disease and future attempts at improving the care of those affected are warranted. The developments of the past 100 years as well as a careful look into the future are presented in this review. 


\section{Geschichte}

\section{$\nabla$}

Die Erstbeschreibung des Asthma bronchiale ist strittig, wohl auch deshalb, weil eine Reihe von Erkrankungen von Asthmaähnliche Beschwerden machen kann. Bereits im alten China, bei den Hebräern und den Römern soll Asthma beschrieben worden sein [1]. Die Stichhaltigkeit dieser Angaben ist fraglich. Hippokra-

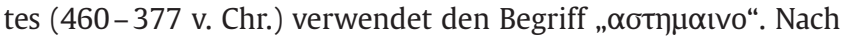
Nolte [2] bezeichnete Homer „Asthma“ als das Keuchen der Griechen beim Kampf um Troja.

Galen (130 - 201 n. Chr. beschrieb den Bronchospasmus und indirekt die Beziehung zwischen oberen und unteren Atemwegen, bei der Körpersäfte aus dem Hirn in die Lunge liefen [1]. Es ist erwähnenswert, dass diese Vorstellung auch heute noch Anhänger findet. Die erste klinische Beschreibung allergischer Rhinitis und von Asthmasymptomen kommt von Sir Charles Blackley, der seine wiederkehrenden saisonalen Symptome als „hayfever“ bezeichnete, die mit thorakaler Enge und nächtlicher Atemnotsymptomatik einhergingen. Die Erfindung des Stethoskops durch Laennec war möglicherweise der erste entscheidende Schritt zu einer gezielten Diagnostik. In der Folgezeit wird zwar zwischen Asthma cardiale und Asthma bronchiale unterschieden, dennoch ist Asthma bronchiale bis in die Mitte des 20. Jahrhunderts eher eine seltene Erkrankung. Als Ursachen werden eine „uracemie respiratoire“ auf dem Boden einer arthritischen Diathese einer „colloidoclassische Diathese“ gegenübergestellt [1]. Sir William Osler beschrieb erstmals, dass „unspezifische“ Atemwegsreize anfallsartige Atemnotanfälle auslösen können und liefert damit wohl die erste Beschreibung der bronchialen Hyperreagibilität. Er hielt Asthma für eine Entzündungskrankheit, wozu ihn der Nachweis der von Charcot und von Leyden beschriebenen Kristalle und der Nachweis der eosinophilen Granulozyten durch die Färbemethoden von Paul Ehrlich bewegten.

Curry ließ 1946 Histamin von Normalpersonen und Asthmapatienten inhalieren [1] und legte damit den Grundstein für eine Untersuchung, die heute zur Diagnostik eines Asthma bronchiale obligat ist. Dennoch wird aus Mangel an pathophysiologischen Erklärungen und aufgrund der Trends und Moden der Zeit Asthma im ausgehenden 19. und beginnenden 20. Jahrhundert vor allem als neurotisch-psychiatrische Erkrankung erkannt und behandelt, auch wenn erbliche, neuronale, vaskuläre und allergische Mechanismen bereits diskutiert werden. Therapeutisch ist in der Mitte des 19. Jahrhunderts als wirksame Therapie von Asthma immerhin starker Kaffee bekannt, was 1922 durch Samuel Hirsch um Theophyllin ergänzt bzw. präzisiert wird, das allerdings erst einige Jahrzehnte später breiteren therapeutischen Einsatz findet.

\section{Vorstellungen zur Pathogenese \\ $\nabla$}

Ein wesentlicher Meilenstein im Verständnis zur Pathogenese war 1966 die Entdeckung des IgEs und seiner Interaktion mit den von Paul Ehrlich beschriebenen Mastzellen durch Ishisaka. Bis dahin bestand die Diagnostik der allergischen Verursachung asthmatischer Beschwerden in der inhalativen, zumindest aber konjunktivalen oder nasalen Allergenprovokation. Dabei stand die glatte Muskelzelle als Ursache des Bronchospasmus und dessen Therapie durch $\beta_{2}$-Agonisten im Vordergrund. IgE und die Mastzelle rückten nun für Jahre in den Fokus des wissenschaftlichen und des klinischen Interesses. Asthma wurde zur Erkrankung der Mastzelle und „Mastzell-Stabilisatoren“ waren Medika- mente der Zeit. Inhalative Glukokortikosteroide wurden erst entwickelt und deren Nebenwirkungen waren noch aus der Zeit, als ausschließlich systemische Glukokortikoide zur Verfügung standen, gefürchtet. Vernachlässigt wurde, dass bereits Jahre zuvor, erstmals in großer klinischer Präzision und Ausführlichkeit, ein nicht-allergischer Phänotyp des Asthmas beschrieben worden war, den Rackemann in seinen Arbeiten als „intrinsic asthma“ bezeichnete. Die Begeisterung für die Rolle der Mastzelle und ihrer Mediatoren, insbesondere des Histamins, blieb ungebrochen, auch als zunehmend klar wurde, dass Histamin-Rezeptor-Antagonisten nicht in der Lage waren, asthmatische Beschwerden zu beeinflussen und darüber hinaus nicht alle Formen des Asthmas auf allergische Mechanismen zurückzuführen sind [3].

Damit folgt auch die Wissenschaft zur Asthmapathogenese den Vorstellungen Thomas Kuhns, der wissenschaftlichen Fortschritt nicht als kontinuierlich, sondern in "Revolutionen“ beschrieb; zwischen den „Revolutionen“, bei denen ein neues Dogma die Oberhand gewinnt - in diesem Fall die Bedeutung der Mastzelle -, kommt es zu einer Phase der Stagnation, bei der es gilt, das geltende Dogma zu untermauern. Untersuchungen, die das geltende Dogma nicht bestätigen - z.B. die Wirkungslosigkeit der Antihistaminika oder der PAF-Antagonisten (siehe unten) -, sind dann keinesfalls geeignet, die geltende Hypothese nach Sir Karl Popper zu falsifizieren, sondern belegen allenfalls das Unvermögen der jeweiligen Untersucher, vernünftige Forschung zu unternehmen.

\section{Platelet-activating factor - PAF}

Anfang der 1980er-Jahre wurde der Platelet-activating factor PAF identifiziert und als entscheidender Mediator in der Pathogenese des Asthmas beschrieben, denn er war in der Lage, in Tiermodellen eine Reihe asthmatypischer Veränderungen zu verursachen. Dazu gehörten die Induktion einer bronchialen Hyperreagibilität, die Ödembildung, Eosinophilie, etc. Asthma wurde zur PAFitis; aber trotz großer Euphorie, dass damit die Ursache von Asthma gefunden wurde, ließ sich PAF beim Asthma des Menschen nie überzeugend nachweisen; hoch wirksame PAF-Antagonisten blieben klinisch völlig wirkungslos [4].

Die Untersuchungen zur Pathogenese des Asthmas werden seit Mitte des 20. Jahrhunderts durch technische Entwicklungen auf interventionellem und immunologischem Gebiet begünstigt. Parallel dazu verlagern sich die Grundlagen medizinisch-wissenschaftlicher Erkenntnis von der persönlichen Beobachtung zu randomisierten klinischen Studien. Biopsiestudien aus starrer und flexibler Endoskopie erlaubten, in vivo endobronchiale Entzündung mit Hilfe immunologischer Färbetechniken zu charakterisieren.

Die Immunologen Mossman und Coffman beschrieben bei allergisierten Mäusen eine T-Helfer-Zell-Subpopulationen, die sie TH-2-Typ-Zellen nannten und für die Pathogenese allergischer Entzündung verantwortlich machten, denn diese Zellen waren in der Lage zwei Zytokine zu bilden, die für die Pathogenese des Asthmas der Maus entscheidend waren: Interleukin-4, das für die IgE Bildung benötigt wird, und Interleukin-5, das die Eosinophilie propagiert. Die Hypothese, dass Asthma eine Erkrankung der TH-2-Zellen und der damit verbundenen Mechanismen darstellt, wurde vorbehaltlos auf das allergische Asthma übertragen. Die Möglichkeit, Zytokine mittels ELISA und anderer Verfahren zu messen oder zumindest deren Transkriptionsmöglichkeit mittels mRNA Nachweis zu führen, untermauerte diese Vorstellungen zur Pathogenese und die sogenannte TH-2-Hypothese wurde in den Folgejahren - in Übereinstimmung mit der Wissenschafts- 
theorie von T. Kuhn - in einer Vielzahl von Untersuchungen bestätigt, obwohl sich rasch zeigen ließ, dass Asthma nicht immer mit einer erhöhten Konzentration z.B. mit Interleukin-4 einhergehen muss [5]. Neuere Untersuchungen der letzten Jahre weisen auf eine geringfügige Beeinflussbarkeit asthmatischer Beschwerden durch anti-Interleukin-4- [6], anti-Interleukin-5- [7] oder anti-Interleukin-13-Strategien hin. Dennoch bleibt deren Wirkung bislang hinter den postulierten Erwartungen zurück.

Airway remodelling durch Hypertrophy der glatten Atemwegsmuskulatur wurde 1973 von Ellul-Micallef beschrieben [8]; Laitinen u. Mitarb. konnten an Biopsien, die sie mit der starren Endoskopie entnahmen, Veränderungen der Atemwegsmatrix bei Asthma zeigen [9]. Aus diesen und darauf folgenden Untersuchungen zur Immunohistopathologie bzw. Immunozytopathologie bei verschiedenen Altersgruppen ergaben sich zwei Konzepte zur Pathogenese des Asthma bronchiale. Das eine geht von einer linearen Pathologie aus, bei der die strukturellen Veränderungen der Atemwege als Folge der Entzündung entstehen, während das andere davon ausgeht, dass das Remodelling der Atemwege, insbesondere die Verdickung der Basalmembran und die Hypertrophie der glatten Atemwegsmuskulatur asthmatypische Prozesse sind, die unabhängig von der Atemwegsentzündung verlaufen. Ob strukturelle Veränderungen der Atemwege, wie z.B. die Verdickung der Basalmembran, sogar einen protektiven Effekt auf die Atemwegskonstriktion ausüben oder eher zum progredienten Lungenfunktionsverlust beim Asthma beitragen, wird diskutiert. Neben den Vorstellungen zum strukturellen Remodelling der Atemwegsarchitektur sind in den letzten Jahren neuronale Mechanismen in den Fokus wissenschaftlicher Untersuchungen zurückgekehrt. So ließen sich erhöhte Konzentrationen an Neurotrophinen in den Atemwegen von Patienten mit Asthma nachweisen [10], die endobronchial nach Allergenprovokation freigesetzt werden [11]. Interventionelle Studien, die mittels Thermoplastie endobronchial Atemwegsstrukturen koagulierten, konnten die Zahl der Exazerbationen reduzieren [12]. Der Mechanismus dieser Technik ist ungewiss, kann aber neben der glatten Muskulatur auch die endobronchiale Innervation einbeziehen.

Weil bis heute keine der erwähnten oder verwandten Hypothesen die Pathogenese des Asthma bronchiale befriedigend erklärt, findet die klinische Beobachtung unterschiedlicher Phänotypen und die Vorstellung, dass durch deren exaktere Definition erfolgreichere Therapieansätze resultieren könnten, neues Interesse. Dabei ist aber weitgehend unklar, inwieweit die beschriebenen Phänotypen Manifestation unterschiedlicher Schweregrade oder Verläufe oder in der Tat eine eigenständige Pathogenese reflektieren.

\section{Phänotypen \\ $\nabla$}

Klinisch wurden in der Vergangenheit verschiedene Phänotypen identifiziert. Das allen gemeinsame diagnostische Vorgehen bei Erwachsenen zeigt schematisch $\bullet$ Abb. 1.

\section{Allergisches Asthma}

Das allergische Asthma manifestiert sich meist im Kindes- und Jugendalter und hat in dieser Altergruppe eine hohe Prävalenz. Patienten mit allergischem Asthma bronchiale haben meist eine positive Familienanamnese für Asthma und andere allergische Erkrankungen wie die allergische Rhinitis oder eine atopische Dermatitis. Häufigste Allergene sind Gräser- und Bäumepollen, Hausstaubmilbenallergen und Haustiere. Neben Sensibilisierun- gen gegen die üblichen inhalativen Umweltallergene muss eine berufliche Allergenexposition in Betracht gezogen werden.

\section{Intrinsic Asthma}

Neben dem allergischen Asthma, dessen Prävalenz sicher vorherrschend ist, lässt sich eine Untergruppe an Patienten mit Asthma identifizieren, deren Beschwerden meist in der zweiten Lebenshälfte beginnen, was gelegentlich als „adult-onset“-Asthma beschrieben wird. Als Auslöser werden oft virale Infektionen der oberen Atemwege angegeben. Diese Patienten weisen keine Sensibilisierungen auf, selbst bei ausführlichen Hauttests. Ihr Gesamt-IgE liegt daher im Normbereich und auch spezifische IgEAntikörper lassen sich nicht nachweisen. Nun ist nicht alles Asthma, das im Erwachsenenalter beginnt, vom intrinsisch/nicht-allergischen Typ, aber in dieser Altergruppe möglicherweise häufiger als das allergische Asthma [13]. Aber auch Kinder können an nicht-allergischem Asthma leiden [14]. Ob das Intrinsic Asthma eine Vorstufe des Churg-Strauss-Syndroms darstellt, wird diskutiert, würde dann aber nur bei einer Minderzahl der Betroffenen tatsächlich auftreten.

\section{Gemischtförmiges Asthma}

Neben dem rein allergischen Asthma und dem Intrinsic Asthma gibt es Patienten, deren Beschwerden in der Kindheit und Jugend durch Allergenexposition ausgelöst wurden. Im Verlauf der Erkrankung ändert sich die Symptomatik jedoch dahingehend, dass allergische Auslöser ihre klinische Bedeutung verlieren, obwohl die kutane Sensibilisierung fortbesteht. Die klinische Reaktion bei Allergenkontakt spielt allerdings kaum mehr eine Rolle und der Verlauf der Erkrankung ähnelt dem eines intrinsischen Asthmas; daraus entstammt der Begriff des „gemischt-förmigen Asthmas [15]. 1958 beschrieb Ogilvie [16] bei diesen Patienten eine „charakteristische Anamnese mit intermittierendem Asthma seit Kindheit, das auf einmal durch persistierenden Husten und Sputum“ kompliziert wird und bei dem anhaltendes Giemen und Atemnot die früheren intermittierenden Asthma-Anfälle ersetzt und „wiederkehrende Atemwegsinfekte während der Wintermonate“ eine zusätzliche Komplikation darstellten [16].

Der Begriff des „Infekt-Asthma“, der oft verwendet wird, ist obsolet, da jede Form des Asthmas im Rahmen eines Infekts exazerbieren kann. Als Grund dafür wird ein immunologischer Defekt angenommen, der zu einer unzureichenden Produktion von Interferonen als Antwort auf den Infekt führt [17].

\section{Brittle Asthma}

Hierbei handelt es sich um eine seltene, schwere Form des Asthmas, an der 0,05\% aller Asthmapatienten leiden sollen [18]. Betroffen sind vor allem Erwachsene, bei denen man zwei Formen unterscheidet:

- Brittle Asthma Typ I:

- Anhaltende und chaotische (>40\%) Variabilität des PEF an mehr als $50 \%$ der Tage über eine Periode von $>6$ Monaten trotz adäquater anti-entzündlicher Therapie (>1,5 mg BDP)

- Instabiles Asthma, das oft auf inadäquate Auslöser reagiert

- > weibliche Patienten (1:2,5 M:F) (18-55 Jahre) mit positivem Haut-Prick-Test und/oder Nahrungsmittelintoleranzen

- oft mit Persönlichkeitsstörungen assoziiert

- hohe Morbidität, häufige Krankenhausaufenthalte 


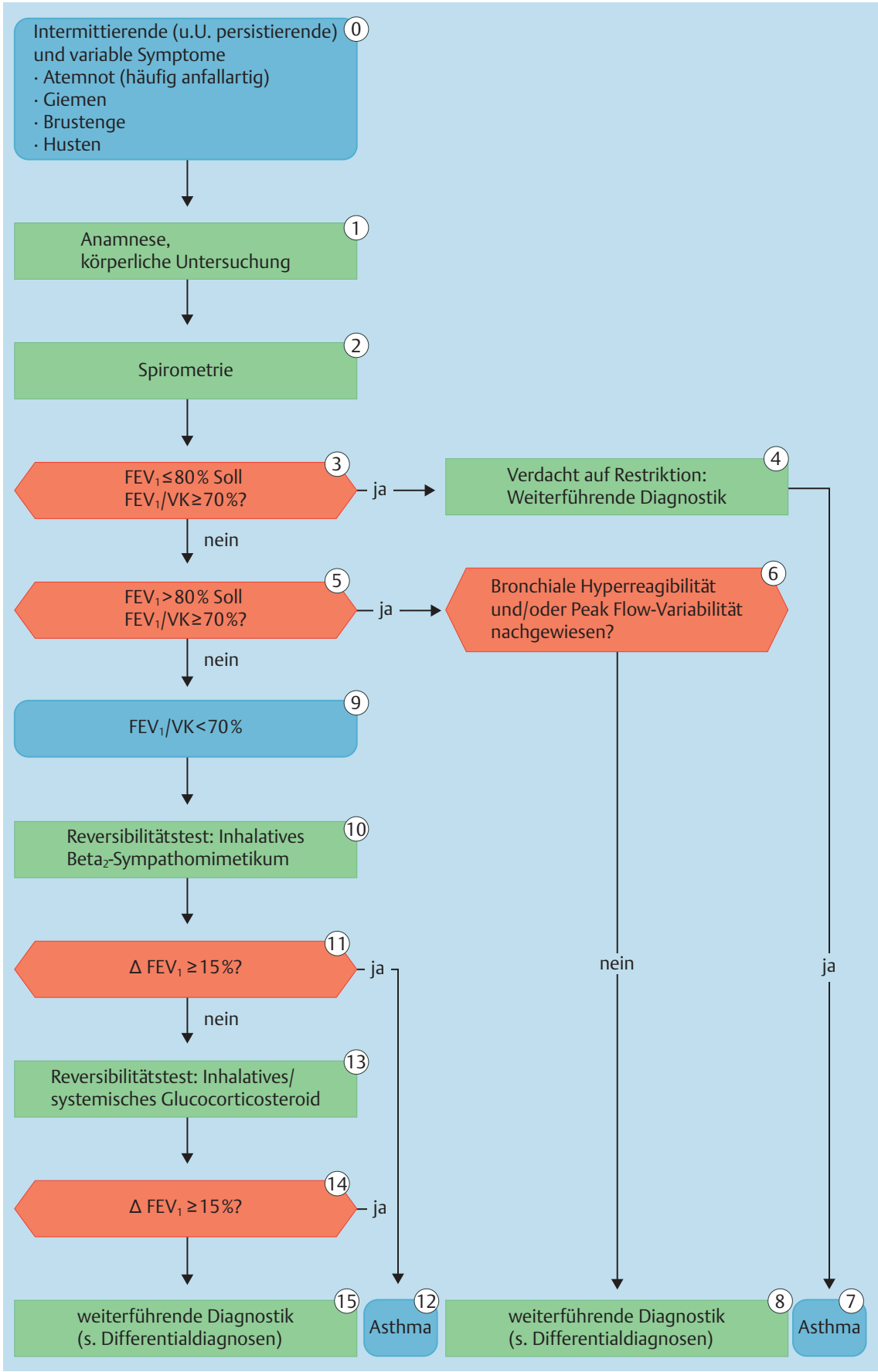

Abb. 1 Algorithmus zur Asthma-Diagnostik bei Erwachsenen; aus [44].

Brittle Asthma Typ II:

- akute schwere Anfälle, die innerhalb von < 3 Stunden ohne erkennbare Auslöser auftreten und zu Notfallbehandlung, mechanischer Beatmung führen und/oder tödlich enden

- vor dem Hintergrund einer normalen oder fast normalen Lungenfunktion bzw. einem gut kontrollierten Asthma auftreten

- ohne Bevorzugung eines Geschlechts (1:1 M:F)

- gehäuft mit Nahrungsmittelallergien einhergehend

Beide Formen des Brittle Asthmas sind mit erhöhter psychiatrischer Morbidität assoziiert: Mehr als die Hälfte der untersuchten Patienten soll eine psychiatrische Anamnese aufweisen und ca. ein Drittel Angststörungen haben [19], was dazu beitragen kann, dass Patienten mit Brittle Asthma dazu neigen, medizinische Hilfe erst verzögert aufzusuchen.

\section{Berufsbedingtes Asthma bronchiale}

Berufsbedingtes Asthma bronchiale wird in Lehrbüchern gerne als eigenständiger Phänotyp geführt, auch wenn sich anamnestisch und klinisch ein klarer Bezug zu exogenen, inhalativen Allergenen oder zumindest Antigenen am Arbeitsplatz zeigen lässt. Spezifische IgE-Antikörper lassen sich nicht gegen alle berufsbedingten Antigene (z. B. Isocyanate) nachweisen, was unterstreicht, dass zirkulierende IgE-Antikörper keine unabdingbare Voraussetzung für die Pathogenese eines Asthmas darstellen.

\section{Anstrengungsasthma - anstrengungsinduzierte \\ Bronchokonstriktion}

Ein weiterer Begriff, der gerne, aber fälschlicherweise zur Klassifikation von Asthma verwendet wurde, ist der des Anstrengungsasthmas. Hierbei handelt es sich nicht um eine Sonderform, son- 
dern um eine Reaktion der hyperreagiblen Atemwege auf Änderungen der Temperatur und der osmotischen Verhältnisse. Anstrengungsasthma, das daher besser als Anstrengungs-induzierte Bronchokonstriktion bezeichnet werden sollte, tritt bei allen Asthmaformen auf, seien sie allergischer oder intrinsischer Genese. Es wird bei Kindern allenfalls häufiger beobachtet und kann in dieser Altergruppe klinisch oft die erste und/oder einzige Manifestation eines beginnenden Asthmas sein.

\section{Analgetika-Asthma}

Eine Untergruppe von Patienten mit vorzugsweise, aber nicht ausschließlich intrinsischem Asthma (ca. 30\% der Betroffenen hat ein allergisches Asthma bronchiale) entwickelt nach Einnahme von nicht-steroidalen Antiphlogistika (NSAID - non-steroidal anti-inflammatory drugs) schwere, therapierefraktäre bronchospastische Krisen. Es handelt sich hierbei um eine akute Verschlechterung einer vorbestehenden, oft schweren Atemwegserkrankung, sodass der Begriff Analgetika-Asthma-Syndrom sicher zutreffender ist als das früher verwendete „Aspirin-induzierte Asthma“. Um der Tatsache Rechnung zu tragen, dass das Asthma fast immer vorbestehend und auch ohne Einnahme von Aspirin therapiebedürftig ist, wurde der Begriff AERD - Aspirin exacerbated respiratory disease vorgeschlagen, für den es noch keine deutsche Entsprechung gibt. „Aspirin-exazerbierte Atemwegserkrankung“ wäre sinnvoll und berücksichtigt, dass eine Anzahl Patienten zunächst nur mit nasaler Symptomatik (v.a. profuser Rhinorrhoe) nach Einnahme von Acetylsalicylsäure, Metamizol, Indomethacin, Piroxicam, Ibuprofen, Diclofenac u.a. reagiert. Gemeinsam ist diesen Medikamenten (die Liste ist nicht vollständig), dass sie die Cyclooxygenase hemmen, wodurch vermehrt Substrat für die 5-Lipoxygenase und damit die Synthese der Leukotriene zur Verfügung steht [20].

Eine spezifische Therapie dieser Erkrankung ist die „adaptive Desaktivierung“, mit der sich durch steigende, niedrige Dosen von Aspirin innerhalb weniger Tage eine Toleranz für therapeutische Dosen induzieren lässt. Ihre Einleitung ist erfahrenen Zentren vorbehalten, da schwere bronchospastische Krisen auftreten können. Die nachfolgende Dauertherapie mit Aspirin in Dosen $\geq 300 \mathrm{mg}$ kann die nasalen und thorakalen Beschwerden bessern. Nach Absetzen tritt das Vollbild der Unverträglichkeit innerhalb weniger Tage wieder auf.

\section{Cluster Analysis}

Die aktuelle Entwicklung zur besseren Klassifikation asthmatischer Phänotypen ist die Analyse sogenannter Cluster. Dabei lässt sich durch computergestützte Analysen Asthma nach Charakteristika klassifizieren, aus denen therapeutische Konsequenzen zu ziehen sind [21]. Operativ lassen sich diese Erkenntnisse, die klinisch durchaus bekannt waren, dahingehend darstellen, dass Asthma nach einem ABC-Schema einzuteilen ist:

A - Atemwegshyperreagibilität - Obstruktion

B - Bronchitis - Entzündung

- FENO

C - Co-Morbiditäten

- Rhinitis, psychosoziale Faktoren

D - Dysfunktionelle Atmung

- VCD, Hyperventilation

E - Extrapulmonale Ursachen

- Adipositas, Rauchen, Allergenexposition, etc.

wobei sich für A und B eine Therapiemöglichkeit mit anti-entzündlicher Therapie ergibt, C-E hingegen unter einer hoch- dosierten Kortikosteroidtherapie weiter verschlechtern können und andere Therapieansätze erfordern.

\section{Asthma bronchiale und chronische Bronchitis/COPD}

Während in den 60er-Jahren des letzten Jahrhunderts die Vorstellung propagiert wurde, dass alle obstruktiven Atemwegserkrankungen in einer gemeinsamen Endstrecke münden [22], ließen sich in den letzten Jahren charakteristische Unterschiede in der Immunpathogenese des Asthmas und der chronischen Bronchitis zeigen, die eine gemeinsame Pathogenese unwahrscheinlich machen. Dennoch finden sich im klinischen Alltag reichlich Patienten, deren Asthma durch langjähriges Inhalationsrauchen kompliziert ist und bei denen eine Abgrenzung zwischen Asthma und COPD problematisch ist. Dies unter anderem deshalb, weil Patienten mit Asthma und chronischem Inhalationsrauchen offenbar rasch dazu neigen, unzureichend auf antiasthmatische Therapie, insbesondere inhalative Glukokortikosteroide anzusprechen. Auf diesem Gebiet der Differenzierung von Asthma und COPD besteht daher unvermindert Forschungsbedarf. Das betrifft besonders die sogenannten „Mischformen“, deren prozentualer Anteil an allen Patienten mit Asthma je nach Autor unterschiedlich hoch angegeben wird, aber bis $\mathrm{zu} 1 / 3$ betragen soll [23]. Hinweise auf die Differenzierung zwischen Asthma und COPD gibt die $\bullet$ Tab. 1, die weiteren zahlreichen Differenzialdiagnosen, die gegen Asthma abzugrenzen sind, sind in - Tab. 2 aufgeführt.

\section{Therapie}

Aus Mangel an wirksamer Pharmakotherapie wurde Asthma in der Vergangenheit mit life-style-Änderungen, Anticholinergikahaltigen, pflanzlichen Extrakten, mit chirurgischer Durchtrennung des Vagus-Nervs, Hypnose und Allergenkarenz therapiert. Obwohl einzelne dieser Methoden immer noch Anhänger finden, ist ihre Wirkungslosigkeit gut dokumentiert. Selbst die strikte Allergenkarenz wird heute als Therapie nicht mehr empfohlen (was nicht mit der Meidung von Allergenen zur Anfallsprophylaxe verwechselt werden darf).

\section{Pharmakotherapie}

Bis in die Mitte des 20. Jahrhunderts fußte die Therapie des Asthma, wie der meisten anderen Erkrankungen, auf empirischen Grundlagen und randomisierte, kontrollierte klinische Studien wurden erst später entwickelt.

Aufgrund der Tatsache, dass man Asthma zu Beginn des 20. Jahrhunderts für eine neurotische oder zumindest psychosomatische Erkrankung hielt, wurden selbst Psychopharmaka wie Chlorpromazin zur Behandlung untersucht, um die „emotionalen Spannungen beim Asthma zu vermindern“.

Asthma-Zigaretten waren wohl die ersten inhalativen Medikamente gegen Asthma. Sie werden heute nur noch als Kuriosum demonstriert, sie enthielten unter anderem Atropin, Belladonna, Menthol, Morphine oder Kokain. Deren tatsächliche Verbreitung und Wirksamkeit wurde aber nie in kontrollierten Studien überprüft. Ein gewisser „healthy survivor“-Effekt ist bei dieser Therapie anzunehmen, bei der nur die Patienten „Asthma-Zigaretten“ beibehielten, die diese Therapie tolerieren konnten. Andererseits ist der Prozentsatz der Patienten mit Asthma, die rauchen, vergleichbar mit der Zahl der Raucher in der Gesamtbevölkerung, was nahelegt, dass Asthmapatienten Zigarettenrauch nicht häufiger meiden als Normalpersonen. 
Tab. 1 Differenzialdiagnose Asthma versus COPD.

\begin{tabular}{|c|c|c|}
\hline Merkmal & Asthma & COPD \\
\hline Alter bei & variabel, häufig: & meist nicht vor der \\
\hline Erstdiagnose & Kindheit, Jugend & 6. Lebensdekade \\
\hline Tabakrauchen & $\begin{array}{l}\text { kein direkter Kausal- } \\
\text { zusammenhang; Ver- } \\
\text { schlechterung durch } \\
\text { Tabakrauchen möglich }\end{array}$ & $\begin{array}{l}\text { direkter Kausal- } \\
\text { zusammenhang }\end{array}$ \\
\hline Hauptbeschwerden & $\begin{array}{l}\text { anfallsartig auftre- } \\
\text { tende Atemnot }\end{array}$ & $\begin{array}{l}\text { Atemnot bei } \\
\text { Belastung }\end{array}$ \\
\hline Verlauf & variabel, episodisch & progredient \\
\hline Allergie & häufig & $\begin{array}{l}\text { kein direkter Kau- } \\
\text { salzusammenhang }\end{array}$ \\
\hline Obstruktion & variabel & persistierend \\
\hline $\begin{array}{l}\text { Reversibilität der } \\
\text { Obstruktion }\end{array}$ & $\Delta \mathrm{FEV}_{1}>15 \%$ & $\Delta \mathrm{FEV}_{1}<15 \%$ \\
\hline $\begin{array}{l}\text { bronchiale } \\
\text { Hyperreaktivität }\end{array}$ & regelhaft vorhanden & möglich \\
\hline $\begin{array}{l}\text { Ansprechen auf } \\
\text { Glucokortikosteroide }\end{array}$ & regelhaft vorhanden & gelegentlich \\
\hline
\end{tabular}

Tab. 2 Differenzialdiagnosen des Asthmas (in alphabetischer Reihenfolge).

\begin{tabular}{l} 
Aspiration (z. B. Fremdkörper) \\
Bronchiektasen \\
chronisch obstruktive Bronchitis mit/ohne Lungenemphysem (COPD) \\
chronisch persistierender Husten \\
diffuse parenchymatöse Lungenerkrankungen (u. a. exogen allergische \\
Alveolitis) \\
Entwicklungsstörung \\
gastroösophagealer Reflux \\
Hyperventilations-Syndrom \\
Immundefekte \\
kardiovaskuläre Erkrankungen und Anomalien (z. B. linksventrikuläre \\
Funktionsstörung, „Pulmonalisschlinge“) \\
\hline Kehlkopfveränderungen \\
Lungenarterienembolie \\
neuromuskuläre Erkrankungen \\
postinfektiöse Störungen \\
primäre Zilien-Funktionsstörung \\
Spontanpneumothorax \\
Stimmbanddysfunktion \\
\hline Tracheo-Bronchomalazie \\
Tuberkulose \\
Tumor (Larynx, Trachea, Lunge) \\
zystische Fibrose \\
\hline
\end{tabular}

\section{Bronchospasmolytische Therapie}

Mitte des 19. Jahrhunderts wurden starker Kaffee und Tee zur Asthmabehandlung eingesetzt. Samuel Hirsch synthetisierte daraus 1922 das Theophyllin [24], das zunächst aber vor allem als Diuretikum Einsatz fand. Dennoch blieb Theophyllin bzw. Aminophyllin der wirksamste Bronchodilatator über Jahrzehnte und verlor erst in den 1990ern hinter inhalativen Glukokortikoiden, $\beta_{2}$-Agonisten und Leukotrien-Antagonisten seine Bedeutung. Als Weiterentwicklung des unspezifischen Phosphodiesterase-Inhibitors Theophyllin wurden in den letzten Jahrzehnten selektive PDE-Inhibitoren entwickelt, deren Vorteil gegenüber Theopyhllin in der Asthmatherapie noch ungewiss ist.

\section{$\beta_{2}$-Agonisten}

Der erfolgreiche Einsatz von Adrenalin und dessen rasche, kurz dauernde und auch nicht nebenwirkungsfreie Bronchodilatation führte zur Entwicklung des Isoproterenols als erstem selektivem, inhalativem $\beta$-Agonisten, der später durch selektive $\beta_{2}$-Agonisten wie Salbutamol, Fenoterol, Terbutalin, Reproterol (in Deutschland in Kombination mit DNCG) und Clenbuterol ersetzt wurde. Salbutamol ist seither der am weitesten verbreitete Bronchodilatator [25]. Ende der 1980er-Jahre kamen Salmeterol und Formoterol auf den Markt, deren Wirkung 12 und mehr Stunden anhielt, ohne dass sie mehr Nebenwirkungen verursachten. Gegenwärtig werden $\beta_{2}$-Agonisten entwickelt, deren Wirkung über 24 Stunden anhält und eine einmal täglich Einnahme erlauben. Indacaterol $\left(\mathrm{Onbrez}^{\circledR}\right)$ ist der erste Vertreter dieser Gruppe, der allerdings bislang nur zur Therapie der COPD zugelassen ist.

Die $\beta_{2}$-Agonisten relaxieren glatte Muskulatur. Aufgrund ihrer raschen bronchodilatierenden Wirkung, die von keiner anderen Medikamentenklasse übertroffen wird, wurde in den 1960ern angenommen, dass Asthma eine Erkrankung der glatten Atemwegsmuskulatur sei. Diese Hypothese wurde in den folgenden Jahrzehnten mit wechselndem Erfolg immer wieder aufgegriffen. Bereits 1969 fiel auf [26], dass der zunehmende Verbrauch an $\beta_{2^{-}}$ Agonisten-Dosieraerosolen mit einer steigenden Mortalität an Asthma einherging, was heute als „Asthma-Paradox“ bezeichnet wird: Der (hochdosierte) Einsatz des wirksamsten Bronchodilatators kann Asthma destabilisieren und zu einem Verlust an Asthmakontrolle führen [27]. Als Mechanismus wurde eine rasch einsetzende Tachyphylaxie identifiziert, bei der die $\beta$-Rezeptoren abnehmen. Darüber hinaus wird diskutiert, dass die bronchodilatierende Wirkung der $\beta_{2}$-Agonisten die Atemwegsentzündung mit ihren Folgen erfolgreich maskieren kann, was eine rechtzeitige anti-entzündliche Intervention verhindert. Hierbei handelt es sich offenbar um ein generelles Prinzip in der Therapie mit Rezeptor-Agonisten, bei der die langfristige bzw. hochdosierte Verwendung zu Wirkungsverlust und unerwünschten Wirkungen führt. Die Vermutung, dass pharmakogenetische Besonderheiten, nämlich Mutationen im $\beta_{2}$-Rezeptor für das individuell unterschiedliche Ansprechen verantwortlich zu machen seien, ließ sich bis dato nicht bestätigen.

\section{Glukokortikosteroide}

Während Asthma bis Anfang der 1950er-Jahre ausschließlich symptomatisch-bronchospasmolytisch behandelt wurde, erlaubte der Einsatz synthetischer Kortikosteroide erstmals Ansätze der Asthmakontrolle. Das von Erstanwendern staunend beschriebene Ansprechen auf die orale oder parenterale Therapie festigte die Vorstellung, dass Asthma eine entzündliche Erkrankung sei. Aufgrund ihrer Nebenwirkungen wurden systemische Glukokortikosteroide jedoch rasch schweren Fällen und der Therapie des akuten Asthmaanfalls vorbehalten. Nicht unerwartet gelang es bis heute nicht, nebenwirkungsärmere oder gar -freie systemische Kortikosteroide zu entwickeln. Rasch erkannte man auch, dass systemische Kortikosteroide die abnehmende Wirkung von $\beta_{2}$-Agonisten nach deren übermäßigem Gebrauch wieder besserten, was man als den „permissiven Effekt“ der Glukokortikosteroide bezeichnete. Als dessen Mechanismus wird bis heute die Stimulation bzw. Wiederherstellung der $\beta$-Rezeptorendichte angenommen.

In den 1970er-Jahren kam mit Beclometason dipropionat (BDP) das erste inhalative Glukokortikosteroid in Gebrauch, das trotz niedriger Dosierung Asthma bei vielen Patienten ohne die üblichen Nebenwirkungen systemischer Glukokortikosteroide deut- 
lich besserte. Als klinisches Maß galt damals, dass sich mit inhalativen Glukokortikosteroiden in normaler Dosierung ca. $10 \mathrm{mg}$ Prednisolonäquivalent und damit dessen Nebenwirkungen einsparen ließen. Dennoch vergingen weitere 20 Jahre, ehe inhalative Glukokortikosteroide (ICS) zur Standardbehandlung des Asthmas wurden. Wesentlich dazu beigetragen haben die fixen Kombinationen von inhalativen Glukokortikosteroiden und $\beta_{2^{-}}$ Agonisten: Mitte der 1980er-Jahre kamen in einigen Ländern erste Kombinationen von Salbutamol und inhalativen Glukokortikosteroiden auf den Markt, die nicht nur eine wesentliche Vereinfachung der Behandlung darstellten, sondern auch die unbalancierte und potenziell nebenwirkungsträchtige Monotherapie mit $\beta_{2}$-Agonisten verhindern konnten. Diese ersten Kombinationen wurden rasch durch Kombinationen langwirksamer $\beta_{2}$-Agonisten und inhalativer Glukokortikosteroide abgelöst, die heute weit verbreitet eingesetzt werden. Auch wenn Sicherheitsstudien darauf hinweisen, dass der dauerhafte Einsatz dieser Kombinationen, insbesondere bei Kindern, mit einem erhöhten Risiko einer Destabilisierung von Asthma einhergehen könnte [28], wird der „permissiven Wirkung“ der Glukokortikosteroide auf die $\beta_{2}$-Agonisten-Wirkung in diesen Kombinationen Rechnung getragen. Sie ist dadurch sicherer als die Monotherapie mit kurz oder lang wirksamen $\beta_{2}$-Agonisten, die heute nicht mehr empfohlen wird.

Die Entwicklung der ICS in den letzten Jahrzehnten war darauf gerichtet, Substanzen mit geringerem Nebenwirkungsprofil (z.B. hohem First-Pass-Effekt oder geringer oraler Bioverfügbarkeit) und stärkerer Wirkung zu finden. Dennoch sind die gegenwärtig verfügbaren inhalativen Glukokortikosteroide in ihrer Wirkung vergleichbar und ohne klinisch unmittelbar feststellbare Unterschiede. Der frühe Einsatz inhalativer Steroide kann progrediente Beschwerden verhindern [29], während sich bis heute keine Hinweise dafür finden, dass sich dadurch eine Protektion vor zukünftigen Asthmabeschwerden erzielen lässt, wenn die Therapie beendet wird [30].

Das heute empfohlene stufenweise, je nach Schweregrad, therapeutische Vorgehen fasst $\bullet$ Tab. 3 zusammen.

\section{Leukotrien-Antagonisten}

Nachdem Samuelson u. Mitarb. als aktiven Bestandteil der „slow reacting substance of anaphylaxis“ die Cysteinyl-Leukotriene charakterisierten, ließ sich zeigen, dass diese verschiedene Charakteristika des Asthma bronchiale vermitteln. Cysteinyl-Leukotriene induzieren bzw. verstärken Bronchokonstriktion und bronchiale Hyperreagibilität, die Akkumulation von Eosinophilen [31] und Atemwegsremodelling. Mit Leukotrien-Rezeptor-Inhibitoren (Cys-LT-1-Rezeptor-Antagonisten: Montelukast, Pranlukast, Zafirlukast) bzw. Synthese-Hemmern (Zileuton) wurde eine neue Gruppe von Medikamenten entwickelt [20,32], die bei leichtem Asthma als Alternative zu niedrig dosierten inhalativen Glukokortikosteroiden (ICS) bzw. bei schwererem Asthma als Zusatz zur Therapie mit ICS mit oder ohne lang wirksame $\beta_{2^{-}}$ Agonisten empfohlen werden [25]. Sie bessern zudem Symptome bei der allergischen Rhinitis und werden zur Behandlung des Analgetika-Asthmas/AERDs empfohlen.

\section{Anticholinergika}

Verschiedene Untersuchungen haben sich ohne überzeugenden Erfolg der Frage gewidmet, ob Anticholinergika antiasthmatische Wirkung besitzen [33]. Die Kombination von Ipatropium bromid mit Fenoterol oder Salbutamol wird seit Jahrzehnten zur Therapie von Asthma und COPD eingesetzt, obwohl die additive Wir-
Tab. 3 Stufentherapie erwachsener Asthma-Patienten.

\begin{tabular}{|c|c|}
\hline \multicolumn{2}{|c|}{ Stufe IV: schwergradig persistierend } \\
\hline Bedarfstherapie: & Dauertherapie: \\
\hline $\begin{array}{l}\text { inhalatives rasch wirksames } \\
\beta_{2} \text {-Sympathomimetikum }\end{array}$ & $\begin{array}{l}\text { ICS in hoher Dosis plus inhalatives lang } \\
\text { wirksames } \beta_{2} \text {-Sympathomimetikum } \\
\text { (ggf. als feste Kombination) und eine oder } \\
\text { mehrere der zusätzlichen Optionen: } \\
\text { - retardiertes Theophyllin } \\
\text { - Omalizumab bei allergischem } \\
\text { Asthma* } \\
\text { - systemisches Glukokortikosteroid } \\
\text { (intermittierend oder dauerhaft) in der } \\
\text { niedrigsten noch effektiven Dosis) }\end{array}$ \\
\hline \multicolumn{2}{|c|}{ Stufe III: mittelgradig persistierend } \\
\hline Bedarfstherapie: & Dauertherapie: \\
\hline $\begin{array}{l}\text { inhalatives rasch wirksames } \\
\beta_{2} \text {-Sympathomimetikum }\end{array}$ & $\begin{array}{l}\text { ICS in niedriger bis mittlerer Dosis plus } \\
\text { inhalatives lang wirksames } \beta_{2} \text {-Sympatho- } \\
\text { mimetikum (ggf. als feste Kombination). } \\
\text { Statt LABA Alternativen, ggf. zusätzliche } \\
\text { Optionen: } \\
\text { - Steigerung der Dosis des } \\
\text { inhalativen Glukokortikosteroids } \\
\text { - Montelukast } \\
\text { - retardiertes Theophyllin } \\
\text { - retardiertes orales } \\
\beta_{2} \text {-Sympathomimetikum }\end{array}$ \\
\hline \multicolumn{2}{|c|}{ Stufe II: geringgradig persistierend } \\
\hline Bedarfstherapie: & Dauertherapie: \\
\hline $\begin{array}{l}\text { inhalatives rasch wirkames } \\
\beta_{2} \text {-Sympathomimetikum }\end{array}$ & $\begin{array}{l}\text { inhalatives Glukokortikosteroid (ICS) } \\
\text { in niedriger Dosis }\end{array}$ \\
\hline \multicolumn{2}{|c|}{ Stufe I: intermittierendes Asthma } \\
\hline Bedarfstherapie: & Dauertherapie: \\
\hline $\begin{array}{l}\text { inhalatives rasch wirksames } \\
\beta_{2} \text {-Sympathomimetikum }\end{array}$ & entfällt \\
\hline
\end{tabular}

kung bzw. die Wirkung des Ipratropium bromids alleine in der Dauertherapie nie überzeugend belegt wurde. Dennoch können einzelne Patienten speziell von Anticholinergika profitieren. Andererseits besitzen Anticholinergika in der Therapie eines schweren Asthmaanfalls additive Wirkung zu $\beta_{2}$-Agonisten. Lang wirksame Anticholinergika wie das Tiotropium wurden bislang beim Asthma wenig untersucht, was die Entwicklung einer zunehmenden Anzahl lang wirksamer Anticholinergika ändern wird.

\section{Cromoglycat und Nedocromil}

In den 1970er-Jahren beherrschten die Mastzelle und ihre Mediatoren die Vorstellungen zur Pathogenese des akuten wie des chronischen Asthmas. Medikamentös wurde versucht, die Mediator-Freisetzung aus Mastzellen mit Substanzen zu unterdrücken, die als „Mastzell-Stabilisatoren“ bezeichnet wurden. Obwohl Cromoglycat als Protagonist dieser Substanzklasse in vitro die Mediatorfreisetzung aus Mastzellen nur zu ca. 10-20\% hemmt und diese Wirkung zudem einer raschen Tachyphylaxie unterliegt [34,35], blieb die Mastzell-Stabilisation die postulierte Wirkung. Klinische Studien, von denen in erster Linie die positiven veröffentlicht wurden, ergaben bei regelmäßigem und hochdosiertem Einsatz geringe Vorteile zu Placebo. Die limitierte Wirkung, die mehrmals täglich erforderliche Anwendung und nicht zuletzt die Erkenntnis, dass Cromoglycat und Nedocromil hinsichtlich ihrer Wirkung selbst niedrig dosierten inhalativen Glukokortikosteroiden unterlegen sind, hat dazu geführt, dass Leitlinien diese Medikamente heute nicht mehr empfehlen. 


\section{Antihistaminika - H1-Antagonisten}

Auf die fehlende Wirkung von Antihistaminika auf Asthmasymptome und Asthma-Kontrolle wurde bereits hingewiesen. Der vorübergehenden Euphorie zur zentralen Rolle der Mastzelle und ihrer Mediatoren in der Pathogenese des Asthmas tat diese Erkenntnis zunächst keinen Abbruch. Auch die Hypothese, dass sich durch vorbeugende Therapie mit Antihistaminika die Entwicklung eines Asthmas bei Risikokindern verhindern lässt, ließ sich nicht bestätigen.

\section{Ketotifen}

Ketotifen wurde als Inhibitor der Mediatorfreisetzung propagiert. Seine Wirkung, einschließlich seiner Nebenwirkungen, entspricht aber in weiten Aspekten denen eines sedierenden Antihistaminikums; es hat keine klinisch relevanten, anti-entzündlichen Eigenschaften. Es wird heute weder zur Therapie allergischer Beschwerden noch zur Asthmatherapie empfohlen.

\section{Omalizumab}

Das erste sogenannte „Biological“ in der Asthmatherapie war Omalizumab, ein humanisierter IgG-Antikörper, der die Konzentration zirkulierender IgE-Antikörper senkt, ohne Mastzellen zu aktivieren. Aufgrund der hohen Kosten wurde Omalizumab nur zur Therapie des schweren, Therapie-refraktären Asthmas zugelassen, obwohl kritisch zu hinterfragen bleibt, ob dessen beste Wirkung nicht insbesondere bei rein allergischen Beschwerden aller Schweregrade anzunehmen sei. In Kombination mit antiasthmatischer Basistherapie ließ sich für Omalizumab eine symptomatische Besserung und die Reduktion inhalativer Glukokortikosteroide zeigen [36,37].

\section{Anti-IL-5 Antikörper}

Verschiedene Studien aus den 1990ern identifizierten Interleukin-5 als Zytokin, das die Eosinophilie des Asthmas reguliert. Erste Untersuchungen mit monoklonalen, anti-IL-5-Antikörpern waren zunächst jedoch enttäuschend [38], vermutlich aufgrund der Wahl falscher Endpunkte. Bei Patienten mit schwerem Asthma bronchiale, deren Eosinophilie auf Glukokortikosteroide unzureichend anspricht, ließ sich jedoch durch den anti-IL-5-Antikörper Mepolizumab eine Reduktion an Exazerbationen zeigen [39]. Auch andere Zytokine wie Interleukin-4 und Interleukin13 [40], aber auch Neurotrophine [41] aktivieren Eosinophile, was die limitierte Wirkung von Mepolizumab erklären könnte.

\section{Spezifische Immuntherapie}

Mit der spezifischen Immuntherapie steht die einzige Therapie allergischer Erkrankungen mit kausalem Ansatz zur Verfügung. Ihre Wirksamkeit bei der allergischen Rhinitis ist gut dokumentiert; inwieweit sie auch bei allergischem Asthma ggf. heilend sein kann, ist unvermindert Gegenstand der Diskussion und laufender Studien. Dass auch Asthmapatienten von einer spezifischen Immuntherapie profitieren, wurde überwiegend aus retrospektiven Subgruppenanalysen von Patienten mit allergischer Rhinitis, die auch an Asthma litten, ermittelt. Danach reduziert die spezifische Immuntherapie bei Patienten mit Asthma Symptome und Medikamentenverbrauch. Erste Untersuchungen bei Kindern weisen darauf hin, dass die spezifische Immuntherapie gegen Gräser- und Bäumepollen die Entwicklung eines Asthmas verhindern kann [42]. Weitere Studien zu dieser Frage sind notwendig. Inwieweit der sublingualen Immuntherapie ähnliche Effekte wie der subkutanen Therapie zukommen, bedarf weiterer Untersuchungen.

\section{Ausblick \\ $\nabla$}

Asthma bronchiale ist unvermindert eine der am weitesten verbreiteten chronischen Erkrankungen mit erheblicher Morbidität und Mortalität und großer sozio-ökonomischer Bedeutung; sie betrifft heute noch vorwiegend den produktiven Teil der Bevölkerung und verursacht damit nicht nur erhebliche primäre (direkte), sondern auch sekundäre (indirekte) Kosten. Dazu gehören die beeinträchtigte schulische Entwicklung, deren Kosten schwer zu messen sind. Vor dem Hintergrund der Asthma-Epidemie der letzten Jahrzehnte und der sich weltweit verschlechternden Qualität der Atemluft, verbunden mit einem sich ändernden Allergenspektrum durch mikro- (Adaptation „westlichen“ Lebensstils) und makroklimatische (Änderung des Allergenspektrums durch globale Erwärmung) ist mit einer weiteren Zunahme weltweit zu rechnen, nach der Asthma zukünftig allenfalls von der Adipositas-Epidemie und ihren Folgekrankheiten (v.a. Diabetes mellitus II) übertroffen wird.

Auch wenn heute behauptet wird, dass ein Großteil der Betroffenen unter der etablierten Therapie weitgehend beschwerdefrei leben könne, wird eine optimale medikamentöse Versorgung aus verschiedenen Gründen oft nicht realisiert [43]. Dazu gehören eine rationale oder irrationale Non-Compliance, vermeintliche oder reale Budgetzwänge und andere Kosten, aber auch verbleibende Restbeschwerden, mangelndes Vertrauen in die empfohlene Therapie und reale oder vermutete Nebenwirkungen. Strategien, die diesen Hindernissen einer optimalen Behandlung entgegenstehen, gilt es weiterzuentwickeln.

Bei der Entwicklung neuer anti-asthmatischer Therapiestrategien setzt neuer Optimismus ein:

Sie umfasst neue inhalative Glukokortikosteroide und lang wirksame $\beta_{2}$-Agonisten, deren Wirkung $24 \mathrm{Std}$. anhält und eine einmal tägliche Gabe erlauben. Inwieweit Dreifach-Kombinationstherapien mit inhalativen Glukokortikosteroiden, lang wirksamen $\beta_{2}$-Agonisten und lang wirksamen Anticholinergika in der Dauertherapie des Asthmas eine Rolle spielen werden, müssen Studien zeigen.

Neue Therapieansätze, wie Chemokin-Antagonisten und monoklonale Antikörper, die die zelluläre Infiltration beeinflussen, werden untersucht. Interleukin-5-Antikörper könnten die Glukokortikosteroid-Wirkung genauso unterstützen wie Interleukin13-Antikörper, die neben der IgE-Bildung auch auf fibrosierende Remodelling-Prozesse eine hemmende Wirkung haben könnten. Dass Neurotrophin-Antagonisten die bronchiale Hyperreagibilität bessern könnten, ist eine attraktive, aber bislang nicht untersuchte Hypothese.

Vor dem Hintergrund weltweit zunehmender respiratorischer Risikofaktoren, die auch die Entwicklung von Asthma begünstigen, insbesondere aber auch vor dem Hintergrund eines zunehmenden Anteils der älteren Bevölkerung weltweit, die ebenfalls an Asthma bzw. dessen Folgezuständen leidet, besteht dringender Bedarf an weltweit verfüg- und finanzierbaren therapeutischen Optionen, aber auch ein ungebrochener Bedarf an einer kurativen Behandlung, deren Ansätze bis heute nicht in Sicht sind. Hingegen ist die Erkenntnis, dass nicht alle Formen und Schweregrade eines Asthmas auf die gegenwärtig verfügbare antiasthmatische Therapie ansprechen, wichtiger Anstoß zur Entwicklung von Therapiestrategien gegen Komorbiditäten und Risikofaktoren, deren Beeinflussungen geeignet sein können, die Morbidität dieser Erkrankung bereits in der Gegenwart zu bessern. 


\section{Interessenkonflikte}

$\nabla$

Der Verfasser ist Referent und Berater fast aller pharmazeutischen Unternehmen, die Medikamente zur Asthmatherapie herstellen.

\section{Literatur}

1 Diamant Z, Boot JD, Virchow JC. Summing up 100 years of asthma. Respir Med 2007; 101: $378-388$

2 Nolte D. Asthma. 7 ed. München: Elsevier, 1998

3 Virchow C. Intrinsic Asthma: Symptomatik, Serum-IgE-Spiegel und Pathophysiologie. Prax Pneumol 1973; 27: 578-591

4 Evans DJ, Barnes PJ, Cluzel $M$ et al. Effects of a potent platelet-activating factor antagonist, SR27417A, on allergen-induced asthmatic responses. Am J Respir Crit Care Med 1997; 156: 11 - 16

5 Walker C, Bode E, Boer L et al. Allergic and nonallergic asthmatics have distinct patterns of T-cell activation and cytokine production in peripheral blood and bronchoalveolar lavage. Am Rev Respir Dis 1992; 146: $109-115$

6 Wenzel S, Wilbraham D, Fuller R et al. Effect of an interleukin-4 variant on late phase asthmatic response to allergen challenge in asthmatic patients: results of two phase 2a studies. Lancet 2007; 370: 1422 1431

7 Haldar P, Brightling CE, Hargadon B et al. Mepolizumab and exacerbations of refractory eosinophilic asthma. N Engl J Med 2009; 360: $973-$ 984

8 Ellul-Micallef R. Airway smooth muscle in health and in asthma. Br J Dis Chest 1973; 67: 107-113

9 Laitinen LA, Heino M, Laitinen A et al. Damage of the airway epithelium and bronchial reactivity in patients with asthma. Am Rev Respir Dis 1985; 131: 599-606

10 Braun A, Lommatzsch $M$, Renz $H$. The role of neurotrophins in allergic bronchial asthma. Clin Exp Allergy 2000; 30: 178-186

11 Virchow JC, Julius P, Lommatzsch $M$ et al. Neurotrophins are increased in bronchoalveolar lavage fluid after segmental allergen provocation. Am J Respir Crit Care Med 1998; 158: 2002 - 2005

12 Castro M, Rubin AS, Laviolette $M$ et al. Effectiveness and safety of bronchial thermoplasty in the treatment of severe asthma: a multicenter, randomized, double-blind, sham-controlled clinical trial. Am J Respir Crit Care Med 2010; 181: 116-124

13 Ross I. Bronchial asthma in Malaysia. Br J Dis Chest 1984; 78: 369- 375

14 Ulrik CS, Backer V, Dirksen A et al. Extrinsic and intrinsic asthma from childhood to adult age: a 10-yr follow-up. Respir Med 1995; 89: 547 554

15 Walker C, Virchow JC Jr., Bruijnzeel PL et al. T cell subsets and their soluble products regulate eosinophilia in allergic and nonallergic asthma. J Immunol 1991; 146: 1829-1835

16 Ogilvie AG. The treatment of patients with severe asthma and chronic bronchitis. Br J Tuberc Dis Chest 1958; 52: 126-133

17 Contoli M, Message SD, Laza-Stanca V et al. Role of deficient type III interferon-lambda production in asthma exacerbations. Nat Med 2006; 12: $1023-1026$

18 Barnes PJ, Woolcock AJ. Difficult asthma. Eur Respir J 1998; 12: 1209 1218

19 Garden GM, Ayres JG. Psychiatric and social aspects of brittle asthma. Thorax 1993; 48: $501-505$

20 Virchow JC Jr, Kroegel C, Luttmann W, Kortsik C et al. Leukotrien-Rezeptorantagonisten, 5-Lipoxygenase- und 5-Lipoxygenase-aktivierendes Protein (FLAP)-Inhibitoren. Neue Therapieform inder Behandlung des Asthma bronchiale? Allergologie 1996; 19: 3-14

21 Haldar P, Pavord ID, Shaw DE et al. Cluster analysis and clinical asthma phenotypes. Am J Respir Crit Care Med 2008; 178: 218-224

22 Orie NG. The relationship between asthma and chronic obstructive and restrictive pulmonary diseases; lessons from the past century. Ned Tijdschr Geneeskd 2002; 146: 1504-1508
23 Wardman AG, Binns V, Clayden AD et al. The diagnosis and treatment of adults with obstructive airways disease in general practice. $\mathrm{Br} \mathrm{J}$ Dis Chest 1986; 80: 19-26

24 Hirsch S. Klinischer und experimenteller Beitrag zur krampflösenden Wirkung der Purinderivate. Klin Wochenschr 1922; 1: 615-618

25 www.ginasthma.com; Stand: 2007

26 Inman WH, Adelstein AM. Rise and fall of asthma mortality in England and Wales in relation to use of pressurised aerosols. Lancet 1969; 2 : 279-285

27 Hasford J, Virchow JC. Excess mortality in patients with asthma on long-acting beta2-agonists. Eur Respir J 2006; 28: 900 - 902

28 Kramer JM. Balancing the benefits and risks of inhaled long-acting beta-agonists - the influence of values. N Engl J Med 2009; 360: 1592 1595

29 Haahtela T, Jarvinen M, Kava $T$ et al. Comparison of a beta 2-agonist, terbutaline, with an inhaled corticosteroid, budesonide, in newly detected asthma. N Engl J Med 1991; 325: 388 - 392

30 Guilbert TW, Morgan WJ, Zeiger RS et al. Long-term inhaled corticosteroids in preschool children at high risk for asthma. N Engl J Med 2006; 354: $1985-1997$

31 Virchow JC Jr., Faehndrich S, Nassenstein C et al. Effect of a specific cysteinyl leukotriene-receptor 1-antagonist (montelukast) on the transmigration of eosinophils across human umbilical vein endothelial cells. Clin Exp Allergy 2001; 31: 836-844

32 Lane SJ. Leukotriene antagonism in asthma and rhinitis. Respir Med 1998; 92: $795-809$

33 Virchow JC, Lommatzsch M. Anticholinergic agents in asthma. In: Polosa R, Holgate ST, ed. Asthma: Current Strategies: Clinical Publishing 2007: 79-90

34 Church MK, Hiroi J. Inhibition of IgE-dependent histamine release from human dispersed lung mast cells by anti-allergic drugs and salbutamol. Br J Pharmacol 1987; 90: 421 - 429

35 Pearce FL. Effect of nedocromil sodium on mediator release from mast cells. J Allergy Clin Immunol 1993; 92: 155-158

36 Humbert M, Beasley R, Ayres J et al. Benefits of omalizumab as add-on therapy in patients with severe persistent asthma who are inadequately controlled despite best available therapy (GINA 2002 step 4 treatment): INNOVATE. Allergy 2005; 60: 309-316

37 Vignola AM, Humbert $M$, Bousquet $J$ et al. Efficacy and tolerability of anti-immunoglobulin E therapy with omalizumab in patients with concomitant allergic asthma and persistent allergic rhinitis: SOLAR. Allergy 2004; 59: 709-717

38 Leckie MJ. Anti-interleukin-5 monoclonal antibodies: preclinical and clinical evidence in asthma models. Am J Respir Med 2003; 2: 245 259

39 Pavord ID, Haldar P, Bradding P et al. Mepolizumab in refractory eosinophilic asthma. Thorax 2010; 65: 370

40 Luttmann W, Knoechel B, Foerster $M$ et al. Activation of human eosinophils by IL-13. Induction of CD69 surface antigen, its relationship to messenger RNA expression, and promotion of cellular viability. J Immunol 1996; 157: 1678 - 1683

41 Nassenstein C, Braun A, Erpenbeck VJ et al. The neurotrophins nerve growth factor, brain-derived neurotrophic factor, neurotrophin-3, and neurotrophin- 4 are survival and activation factors for eosinophils in patients with allergic bronchial asthma. J Exp Med 2003; 198: 455 467

42 Jacobsen L, Niggemann B, Dreborg $S$ et al. Specific immunotherapy has long-term preventive effect of seasonal and perennial asthma: 10-year follow-up on the PAT study. Allergy 2007; 62: $943-948$

43 Hasford J, Uricher J, Tauscher $M$ et al. Persistence with asthma treatment is low in Germany especially for controller medication - a population based study of 483,051 patients. Allergy 2010; 65: 347-354

44 Buhl R, Berdel D, Criée CP et al. Leitlinie zur Diagnostik und Therapie von Patienten mit Asthma Pneumologie 2006; 60: 139-177 